\title{
Comparison of survival outcomes of neoadjuvant therapy and direct surgery in IB2/IIA2 cervical adenocarcinoma: a retrospective study
}

\author{
Peilin Ouyang ${ }^{1,2} \cdot$ Jingting Cai ${ }^{1} \cdot$ Lin Gui $^{1} \cdot$ Shan Liu $^{1} \cdot$ Na-Yi Yuan Wu ${ }^{1}$. Jing Wang ${ }^{1,2}$
}

Received: 29 August 2019 / Accepted: 12 March 2020 / Published online: 27 March 2020

(c) The Author(s) 2020

\begin{abstract}
Purpose This retrospective study compared the efficacy and survival of patients with cervical adenocarcinoma (IB2/IIA2; FIGO2009) treated with neoadjuvant chemotherapy before radical surgery (NACT + RS), neoadjuvant chemoradiation therapy before radical surgery (NACRT + RS), or primary radical surgery (RS).

Methods Between January 2008 and November 2015, 91 patients diagnosed with stage IB2/IIA2 cervical adenocarcinoma were enrolled, including 29 patients who received RS, 24 patients who received NACT + RS, and 38 patients who received NACRT + RS.

Results The characteristics of patients were balanced among the three groups, and the median follow-up time was 72 months. The 5 year disease-free survival (DFS) rate was $75.8 \%$ and the 5 year overall survival (OS) rate was $85.0 \%$. Univariate analysis revealed that effectiveness of neoadjuvant treatment, tumor size, lymph node metastases, and depth of stromal invasion were the factors predicting recurrence and mortality. Multivariate Cox proportional analysis revealed that the occurrence of a lymph node metastasis was an independent prognostic factor of DFS (hazard ratio $[\mathrm{HR}]=0.223 ; 95 \%$ confidence interval [CI]: 0.060-0.827) and OS (HR $=0.088 ; 95 \% \mathrm{CI}: 0.017-0.470)$. On survival analysis of preoperative adjuvant chemotherapy and primary surgery, the 5 year OS $(P=0.010)$ and DFS $(P=0.016)$ rates for the NACRT + RS group were significantly lower than those for the RS group.

Conclusion Stage IB2/IIA2 cervical adenocarcinoma patients who received primary RS had a better DFS and OS than those who received preoperative NACRT. There was no significant difference when compared to the preoperative NACT group.
\end{abstract}

Keywords Cervical adenocarcinoma $\cdot$ NACT $\cdot$ NACRT $\cdot$ Radical surgery $\cdot$ Survival

\section{Introduction}

There were an estimated 570,000 cervical cancers diagnosed and 311,000 deaths from cervical cancer worldwide in 2018 [1]. The most common cervical histology is squamous cell carcinoma (SCC); cervical adenocarcinoma (AC) accounts for approximately $15-20 \%$ of cervical cancer cases. AC is

Na-Yi Yuan Wu

wunayiyuan@163.com

$\triangle$ Jing Wang

wangjing0081@hnca.org.cn

1 Hunan Cancer Hospital/The Affiliated Cancer Hospital of Xiangya School of Medicine, Central South University, 283, Tongzipo Road, Changsha, Hunan, People's Republic of China

2 Xiangya School of Medicine, Central South University, Changsha, Hunan, People's Republic of China considered to develop within the cervix and has no obvious symptoms in the early stages; therefore, it is usually diagnosed in advanced stage [2]. AC incidence rates have increased in Europe, and Finland, Slovakia, and Slovenia by approximately $3 \%$ in recent decades [3].Similarly, the proportional incidence of cervical $\mathrm{AC}$ has also increased in the United States [4]. A population-based analysis demonstrated that the 5 year survival rates for stage IB2 and IIA SCC were 69 and $58.3 \%$, respectively, whereas those for stage IB2 and IIA AC were 68.3 and $45.5 \%$, respectively, obviously lower than those for SCC [5]. Concurrent chemoradiotherapy (CCRT) is regarded as the primary treatment for locally advanced cervical cancer (LACC) based on benefits of lower regional recurrence and longer survival outcomes compared to radical surgery (RS) [6]. Several studies have pointed out that regardless of treatment (i.e., definitive radiotherapy (RT) or CCRT), the survival benefit for AC is lower than that for SCC $[7,8]$. There are several potential 
reasons for this observation: (1) survival outcomes of $\mathrm{AC}$ may affected by different subtypes, such as gastric type AC, clear cell AC, and endometrial endometrioid AC; (2) AC is not as sensitive to RT as SCC [7, 9, 10], which also results in high rates of locoregional failure [8]; and (3) compared to SCC, AC's biological characteristics are more radical, including higher local recurrence, lymph node metastasis, and distant metastasis rates than SCC [11]. Therefore, it is important to distinguish AC from SCC and formulate more individualized treatment protocols. Studies have shown that preoperative neoadjuvant chemotherapy (NACT) is well tolerated and beneficial in reducing tumor size, can improve long-term disease-free survival (DFS) and overall survival (OS), and the accuracy of frozen section examination in pelvic lymph node operation is not affected [9, 12]. Although multiple studies have found that NACT before RS does not improve OS in patients with local AC when compared to CCRT or RS alone [13-17], whether cervical AC benefits from neoadjuvant therapy is still uncertain. The purpose of this retrospective study was to compare the outcomes of platinum-based NACT followed by radical hysterectomy and radical hysterectomy alone for patients with cervical AC.

\section{Materials and methods}

\section{Patients}

This retrospective study included 91 patients with histologically diagnosed cervical IB2/IIA2 AC who underwent radical hysterectomy with pelvic lymphadenectomy between January 2008 and November 2015 at the Division of Gynecologic Oncology at the affiliated Cancer Hospital of Xiangya School of Medicine. Pre-treatment evaluation included medical history, European Cooperative Oncology Group (ECOG) performance status, gynecologic and physical examination, laboratory exams, tumor biopsy, and chestabdominal-pelvic computed tomography. Patient information was taken from the hospital's case recording system, including the treatment process (NACT regimen, pathological characteristics, and surgical records).

\section{Eligibility}

Inclusion criteria were as follows: (1) adenocarcinoma histologically confirmed by two pathologists based on the World Health Organization staging system for tumors of the uterine cervix; (2) FIGO stages IB2 and IIA2 as determined by two or more gynecologic oncologists. Clinical staging was performed according to the International Federation of Gynecology and Obstetrics staging criteria (FIGO2009); (3) no concomitant malignancy or prior invasive malignancy; (4) at least two cycles of chemotherapy in patients who received NACT; (5) no other serious complications before treatment; and (6) ECOG performance status $\geq 2$. Patients with histologically confirmed SCC, adenosquamous carcinoma, neuroendocrine carcinoma, clear cell carcinoma, and other scarce histologies were excluded from this study.

\section{Ethics approval and consent to participate}

The project was licensed by the Hunan Cancer Hospital ethics committee (project number: 2015[01]) and the Chinese Clinical Trial Registry (registration number: ChiCTR1800018931).

\section{Treatments}

All patients underwent radical hysterectomy type III (Piver-Rutledge classification) [18]. 11 patients received laparoscopy, 80 received laparotomy, and only one patient underwent adnexal preservation. 29 patients received RS directly (RS group) and 24 patients received 2-3 cycles of platinum-based NACT before surgery (NACT + RS group). The following chemotherapy regimens were administered: TP: paclitaxel $135-175 \mathrm{mg} / \mathrm{m}^{2}$ on day 1 and cisplatin $50 \mathrm{mg} / \mathrm{m}^{2}$ on day 2 , repeated every 3 weeks; TC: paclitaxel $135-175 \mathrm{mg} / \mathrm{m}^{2}$ on day 1 and carboplatin area under the curve $5 \mathrm{mg} / \mathrm{mL} / \mathrm{min}$ on day 2 , repeated every 3 weeks; and TN: paclitaxel $135-175 \mathrm{mg} / \mathrm{m}^{2}$ on day 1 and nedaplatin $75-80 \mathrm{mg} / \mathrm{m}^{2}$ on day 2 , repeated every 3 weeks. 38 patients underwent 3-4 cycles of brachytherapy followed by one cycle of platinum-based NACT (NACRT + RS group). ${ }^{192} \mathrm{Ir}$ intracavity brachytherapy was delivered at a dose of $600 \mathrm{cGy}$ at point A twice a week. The indication for adjuvant radiation therapy was the following risk factors: lymph node involvement, compromised surgical margin, parametrial infiltration, and depth of stromal invasion more than half of the cervix. Patients with risk factors received external beam radiation therapy (EBRT) and brachytherapy with concomitant chemotherapy. There were 36 patients who underwent postoperative EBRT because of lymph node involvement or deep stromal invasion: 11 in the NACT + RS group, 13 in the NACRT + RS group, and 12 in the RS group. No patients underwent EBRT because of parametrial infiltration or compromised surgical margin. Patients received postoperative EBRT with total dose of 45 Gy in 23-25 fractions, followed by brachytherapy twice a week for each A point at $600 \mathrm{cGy}$ for a total dose of $42 \mathrm{~Gy}$. During radiotherapy, patients received platinum-based chemotherapy on a 1 week cycle.

\section{Evaluation of short-term response}

Complete response (CR) was defined as complete disappearance of the cervical lesions without lymph node metastasis. Optimal partial response (OPR) was defined as residual 
lesion interstitial infiltration less than $3 \mathrm{~mm}$ with or without lymph node metastasis. Pathological optimal response (pOR) was the total number of cases with CR and OPR. Pathological suboptimal response included persistent residual disease with more than $3 \mathrm{~mm}$ interstitial infiltration in the surgical specimen. The pathological evaluation was performed by two pathologists.

\section{Statistical analysis}

OS was defined as the time from the start of the study to the date of death or the last date the patient was seen. DFS was defined as the date of surgery to the date of recurrence. The date of death was confirmed by the local government or hospital follow-up records. OS and DFS curves were calculated by the Kaplan-Meier method, and statistical differences between each group were evaluated by the log-rank test. Cox proportional hazard models were performed to estimate hazard ratios (HRs). $P$ values less than 0.05 were considered significant. SPSS version 22.0 was used for statistical analysis.

\section{Results}

\section{Patient characteristics}

From January 2008 to November 2015, a total of 91 patients diagnosed with stage IB2/IIA2 cervical AC were eligible for this study (Fig. 1). The patient characteristics are displayed in Table 1 . The mean age was 47 years (range $22-65$ years). There were 24 patients in the NACT + RS group, 38 patients in the NACRT + RS group, and 29 patients in the RS group. 21 patients had lymph node metastasis: 5 patients in the $\mathrm{NACT}+\mathrm{RS}$ group, 10 patients in the NACRT + RS group, and 6 patients in the RS group. 30 patients had deep stromal
Table 1 Patients characteristics

\begin{tabular}{|c|c|c|c|c|}
\hline Item & $\begin{array}{l}\mathrm{NACT}+\mathrm{RS} \\
(n=24) N(\%)\end{array}$ & $\begin{array}{l}\text { NACRT + RS } \\
(n=38) N(\%)\end{array}$ & $\begin{array}{l}\mathrm{RS} \\
(n=29) N(\%)\end{array}$ & $P$ \\
\hline \multicolumn{5}{|l|}{ Age (years) } \\
\hline$<47$ & $17(70.8 \%)$ & $25(65.8 \%)$ & $19(65.5 \%)$ & \multirow[t]{2}{*}{0.899} \\
\hline$\geq 47$ & $7(29.2 \%)$ & $13(34.2 \%)$ & $10(34.5 \%)$ & \\
\hline \multicolumn{5}{|c|}{ Tumor size $(\mathrm{cm})$} \\
\hline$\leq 5$ & $10(41.7 \%)$ & $15(39.5 \%)$ & $17(58.6 \%)$ & \multirow[t]{2}{*}{0.458} \\
\hline$>5$ & $14(58.3 \%)$ & $23(60.5 \%)$ & $12(41.4 \%)$ & \\
\hline \multicolumn{5}{|c|}{ Histological grade } \\
\hline Grade 1 & $9(37.5 \%)$ & $19(50.0 \%)$ & $8(27.6 \%)$ & \multirow[t]{3}{*}{0.413} \\
\hline Grade 2 & $11(45.8 \%)$ & $16(42.1 \%)$ & $16(55.2 \%)$ & \\
\hline Grade 3 & $4(16.7 \%)$ & $3(7.9 \%)$ & $5(17.2 \%)$ & \\
\hline \multicolumn{5}{|c|}{ Lymphnode metastasis } \\
\hline Positive & $5(20.8 \%)$ & $10(26.3 \%)$ & $6(20.7 \%)$ & \multirow[t]{2}{*}{0.825} \\
\hline Negative & $19(79.2 \%)$ & $28(73.7 \%)$ & $23(79.3 \%)$ & \\
\hline \multicolumn{5}{|l|}{ Stage } \\
\hline IB2 & $15(62.5 \%)$ & $29(76.3 \%)$ & $26(89.7 \%)$ & \multirow[t]{2}{*}{0.062} \\
\hline IIA2 & $9(37.5 \%)$ & $9(23.7 \%)$ & $3(10.3 \%)$ & \\
\hline \multicolumn{5}{|c|}{ Depth of muscular invasion } \\
\hline$<1 / 2$ & $14(58.3 \%)$ & $29(76.3 \%)$ & $18(62.1 \%)$ & \multirow[t]{2}{*}{0.269} \\
\hline$\geq 1 / 2$ & $10(41.7 \%)$ & $9(23.7 \%)$ & $11(37.9 \%)$ & \\
\hline
\end{tabular}

$N A C T$ neoadjuvant chemotherapy, NACRT neoadjuvant chemo-radiation therapy, $R S$ radical surgery

invasion: 10 patients in the NACT + RS group, 9 patients in the NACRT + RS group, and 11 patients in the RS group.

\section{Effectiveness and toxicity of neoadjuvant therapy}

Thirty patients who underwent neoadjuvant therapy achieved pOR: 10 in the NACT group (41.7\%) and 20 in the NACRT group (52.6\%) (Table 2). The incidences of myelosuppression, hepatic and renal dysfunction, and sensory neuropathy were similar between the two groups. The
Fig. 1 The group of experiment and the process of treatment

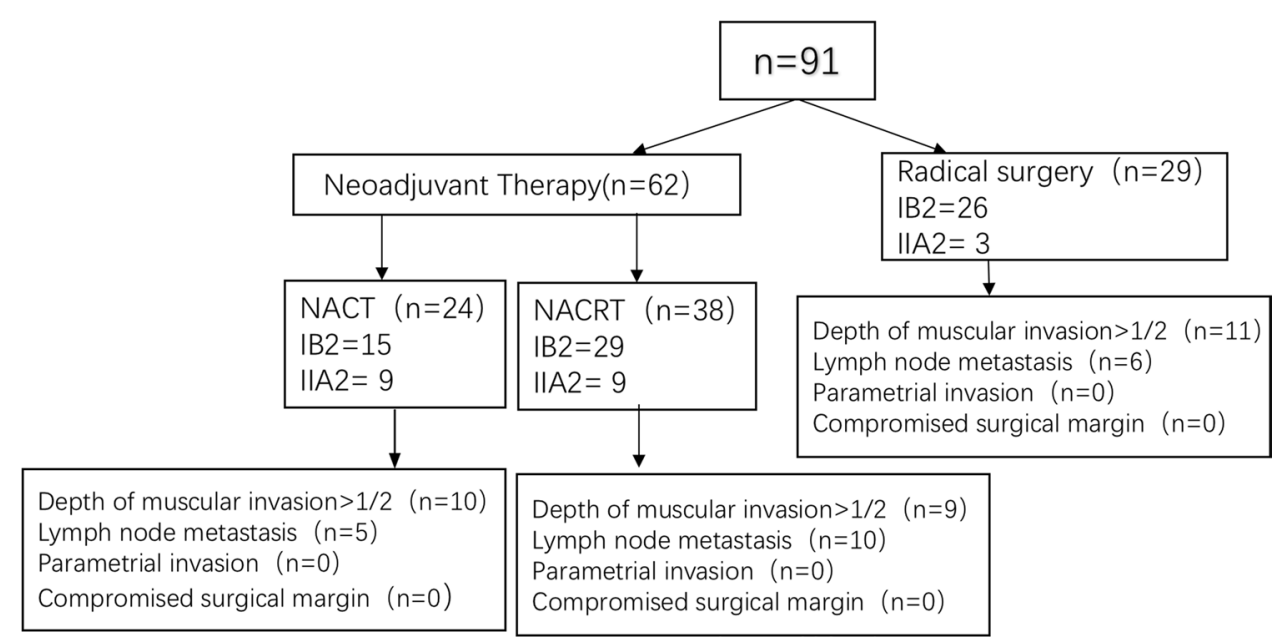


Table 2 Effectiveness of neoadjuvant therapy

\begin{tabular}{lllc}
\hline Terms & CR $(n)$ & OPR $(n)$ & SR $(n)$ \\
\hline NACRT & & & \\
B2 & 6 & 9 & 14 \\
IIA2 & 1 & 4 & 4 \\
NACT & & & \\
IB2 & 1 & 6 & 8 \\
IIA2 & 0 & 3 & 6 \\
\hline
\end{tabular}

NACT neoadjuvant chemotherapy, NACRT neoadjuvant chemo-radiation therapy, $C R$ complete response, $O P R$ optimal partial response, $S R$ suboptimal response

incidence of nausea/vomiting in the NACT group was higher than that in the NACRT group. 1 patient in the NACT group developed grade 3 neutropenia with TP and 2 patients in the NACRT group developed grade 3 neutropenia with TC and $\mathrm{TP}$, respectively. All of these patients had normal neutrophil levels after administration of granulocyte colony-stimulating factor. No patients discontinued neoadjuvant therapy due to therapeutic toxicity (Table 3). The cumulative toxicities of postoperative pelvic EBRT are listed in Table 4. Adverse events of postoperative pelvic EBRT included one case of proctitis in the RS group, two cases of vaginal stenosis and one case of cystitis in the NACRT + RS group, and one case of dermatitis and one case of vaginal stenosis in the NACT group.

\section{Survival}

The 5 year DFS rates for patients undergoing NACT + RS, NACRT + RS, and RS were 73.7, 68.4, and 91.8\%, respectively $(P=0.053)$. The 5 year OS rates were $86.8 \%$ for patients receiving NACT $+\mathrm{RS}, 72.9 \%$ for those receiving NACRT + RS, and $100.0 \%$ for those undergoing RS $(P=0.035)$. There were 21 patients with positive lymph
Table 4 Cumulative toxicity of postoperative pelvic EBRT

\begin{tabular}{llll}
\hline Site & $\begin{array}{l}\text { NACT } \\
(n=11)\end{array}$ & $\begin{array}{l}\text { NACRT } \\
(n=13)\end{array}$ & RS $(n=12)$ \\
\hline Proctitis & 0 & 0 & 1 \\
Cystitis & 0 & 1 & 0 \\
Vaginal stenosis & 1 & 2 & 0 \\
Dermatitis & 1 & 0 & 0
\end{tabular}

$N A C T$ neoadjuvant chemotherapy, $N A C R T$ neoadjuvant chemo-radiation therapy, $R S$ radical surgery

nodes who were treated with postoperative CCRT. The 5 year DFS rate in this group was $37.0 \%$, and the 5 year OS rate was $60.8 \%$ (Table 5). DFS and OS curves by treatment group are shown in Fig. 2. There was no statistically significant difference between the RS group and the NACT + RS group in the 5 year DFS (91.8\% vs $73.7 \%, P=0.222$, Fig. 2a) or the 5 year OS ( $100.0 \%$ vs $82.9 \%, P=0.120$, Fig. $2 b)$. However, the 5 year DFS in the RS group was significantly higher than that in the NACRT + RS group $(91.8 \%$ vs $65.3 \%$, $P=0.016$, Fig. 2c); the same was observed for the 5 year OS (100.0\% vs $72.9 \%, P=0.010$, Fig. 2 d). DFS and OS curves of the patients with pathological optimal response in the neoadjuvant therapy group compared to the RS group are shown in Fig. 3.

\section{Risk factors for recurrence and death}

The results of the univariate analyses are shown in Table 5 . Tumor size, lymph node metastasis, treatment type, and response to neoadjuvant therapy were significant variables for both DFS and OS. Multivariate analysis by Cox proportional hazards model showed that lymph node metastasis was an independent prognostic factor for DFS and OS (Table 6).
Table 3 Summary of Grade $\geq 3$ adverse events

\begin{tabular}{|c|c|c|c|c|c|c|}
\hline \multirow[t]{2}{*}{ Adverse event } & \multicolumn{3}{|c|}{ NACT $(n=24)$} & \multicolumn{3}{|c|}{ NACRT $(n=38)$} \\
\hline & $\mathrm{TC}$ & $\mathrm{TP}$ & $\mathrm{TN}$ & $\mathrm{TC}$ & $\mathrm{TP}$ & $\mathrm{TN}$ \\
\hline & 5 & 6 & 13 & 8 & 18 & 12 \\
\hline Grade 3-4 myelosuppression & 0 & 1 & 0 & 1 & 1 & 0 \\
\hline Grade $3-4$ creatinine & 0 & 0 & 1 & 1 & 1 & 0 \\
\hline Grade 3-4 hypohepatia & 1 & 1 & 1 & 1 & 1 & 0 \\
\hline Nausea/vomiting & 2 & 3 & 2 & 1 & 2 & 0 \\
\hline Sensory neuropathy & 1 & 1 & 1 & 1 & 1 & 1 \\
\hline
\end{tabular}

$N A C T$ neoadjuvant chemotherapy, NACRT neoadjuvant chemo-radiation therapy, TC paclitaxel plus carboplatin, $T P$ paclitaxel plus cisplatin, $T N$ paclitaxel plus nedaplatin 
Table 5 Univariate analysis of disease-free survival and overall survival

\begin{tabular}{|c|c|c|c|c|c|}
\hline Variable & Pts & 5 year DFS $(\%)$ & $P$ value & 5 year OS $(\%)$ & $P$ value \\
\hline \multicolumn{6}{|c|}{ Tumor size $(\mathrm{cm})$} \\
\hline$\leq 4$ & 42 & 83.3 & 0.019 & $90.5 \%$ & 0.003 \\
\hline$>4$ & 49 & 69.1 & & $80.0 \%$ & \\
\hline \multicolumn{6}{|c|}{ Lymphnode metastasis } \\
\hline Negative & 70 & 88.0 & 0.000 & $92.7 \%$ & 0.000 \\
\hline Positive & 21 & 37.0 & & $60.8 \%$ & \\
\hline \multicolumn{6}{|l|}{ Stage } \\
\hline IB2 & 70 & 80.2 & 0.082 & $91.0 \%$ & 0.010 \\
\hline IIA2 & 21 & 61.2 & & $64.0 \%$ & \\
\hline \multicolumn{6}{|c|}{ Depth of muscular invasion } \\
\hline$<1 / 2$ & 61 & 81.1 & 0.005 & $87.9 \%$ & 0.018 \\
\hline$\geq 1 / 2$ & 30 & 65.4 & & $79.4 \%$ & \\
\hline \multicolumn{6}{|c|}{ Effectiveness of neoadjuvant therapy } \\
\hline OR & 30 & 89.7 & 0.001 & $68.5 \%$ & 0.007 \\
\hline SR & 32 & 49.8 & & $88.9 \%$ & \\
\hline \multicolumn{6}{|l|}{ Treatment } \\
\hline NACT & 24 & 73.7 & 0.053 & $86.8 \%$ & 0.035 \\
\hline NACRT & 38 & 68.4 & & $72.9 \%$ & \\
\hline RS & 29 & $91.8 \%$ & & $100 \%$ & \\
\hline
\end{tabular}

NACT neoadjuvant chemotherapy, NACRT neoadjuvant chemo-radiation therapy, $R S$ radical surgery, $D F S$ disease free survival, $O S$ overall survival, $O R$ optimal response, $S R$ suboptimal response

\section{Discussion}

Treatment selection for cervical AC should be based on a comprehensive assessment of resource availability. For the purpose of providing evidence-based, resource-stratified global recommendations to policy makers, ASCO takes into account the function of preoperative chemotherapy in areas where resources are limited [19]. Recently, a phase II study showed that dose-dense neoadjuvant paclitaxel/ carboplatin is feasible and safe in LACC patients. However, it is unclear how neoadjuvant chemotherapy affects survival [20]. The survival benefit of NACT is currently being evaluated in the EROTC 55994 trial, and thus far, they have found no difference in the 5 year OS between patients receiving NACT + RS and those receiving CCRT [21]. Gupta et al. reported that LACC patients treated with $\mathrm{NACT}+\mathrm{RS}$ had inferior DFS compared to patients treated with CCRT $(P=0.003)$; the incidences of adverse events in both groups were within acceptable limits. This prospective study showed a rigorous design and high level of evidence, but they included 179 stage IIB patients who underwent RS, and subgroup analysis showed that the difference was mainly due to these patients [22]. Further, this study included only patients with SCC. As there are differences between AC and SCC in epidemiology, biological characteristics, and chemoradiotherapy sensitivity, the best treatment model for cervical AC is likely not reflected in these clinical trials.

In this retrospective analysis, pathological response criteria were used to evaluate the short-term effects of neoadjuvant therapy. These criteria have been used in many multicenter retrospective analyses and balance the shortage of imaging resources. 10 patients in the NACT $+\mathrm{RS}$ group achieved PR (41.7\%), as did 20 patients in the NACRT + RS group (52.6\%). The pathological response criteria are stricter than the Response Evaluation Criteria in Solid Tumors (RECIST), which define PR as residual lesions $<3 \mathrm{~mm}$ and no lymph node metastasis. For this reason, PR rates are lower when judged by pathological response criteria. The response rate of neoadjuvant chemotherapy when judged by RECIST criteria fluctuates from 48.4 to $93.0 \%$, and in studies assessing response by pathology, the response rate fluctuates from 27.6 to $30.6 \%$ [23, 24]. Several articles also pointed out that pathological response was an indicator for satisfying clinical outcome [16, 25].

Multivariate analysis showed that lymph node metastasis was an independent prognostic factor of survival. The rate of lymph node positivity was $23.1 \%$ in our study, and the 5 year survival rate in patients with lymph node metastasis was $37.0 \%$. Baalbergen et al. found that the survival rate in patients with surgically treated stage I-IIB AC was approximately $91 \%$ if the lymph nodes were negative, but it dropped to $10-34 \%$ if they were positive [26]. Irie et al. found that the incidence of lymph node involvement was significantly higher in patients with $\mathrm{AC}$ than in those with SCC (31.6\% vs $14.8 \%)$ [27]. Mabuchi et al. found that the impact of pelvic nodal metastasis was larger in patients with AC histology than in those with SCC histology (HR: 12.9 versus 3.51), and Cox proportional hazards model indicated a negative response to therapy in AC patients with lymph node metastasis [28].

A number of studies have indicated that AC is not as sensitive as SCC to either radiotherapy or CCRT [10-13]. Yokoi et al. compared the survival outcomes of AC/ASC and SCC patients receiving definitive radiotherapy and found that the 5 year OS rates were 26.7 and $58.6 \%$, respectively, although that study included only 24 AC/ASC patients [7]. One institution in China matched and compared 744 SCC and 71 AC patients who underwent RT or CCRT. They found that patients with $\mathrm{AC}$ were more likely to experience recurrence and had worse survival outcomes than patients with SCC [8]. The smaller number of AC patients may be part of the reason for the difference in survival outcomes. Ryu et al. sought to determine a new criterion that included $\mathrm{AC}$ as an intermediate-risk factor for recurrence and found that it was more sensitive and specific when compared to the Classic model or the GOG model $(P=0.0048)$ [29]. Further, the survival rate of some subtypes of AC may be worse, such as endometrial endometrioid adenocarcinoma, gastric type 

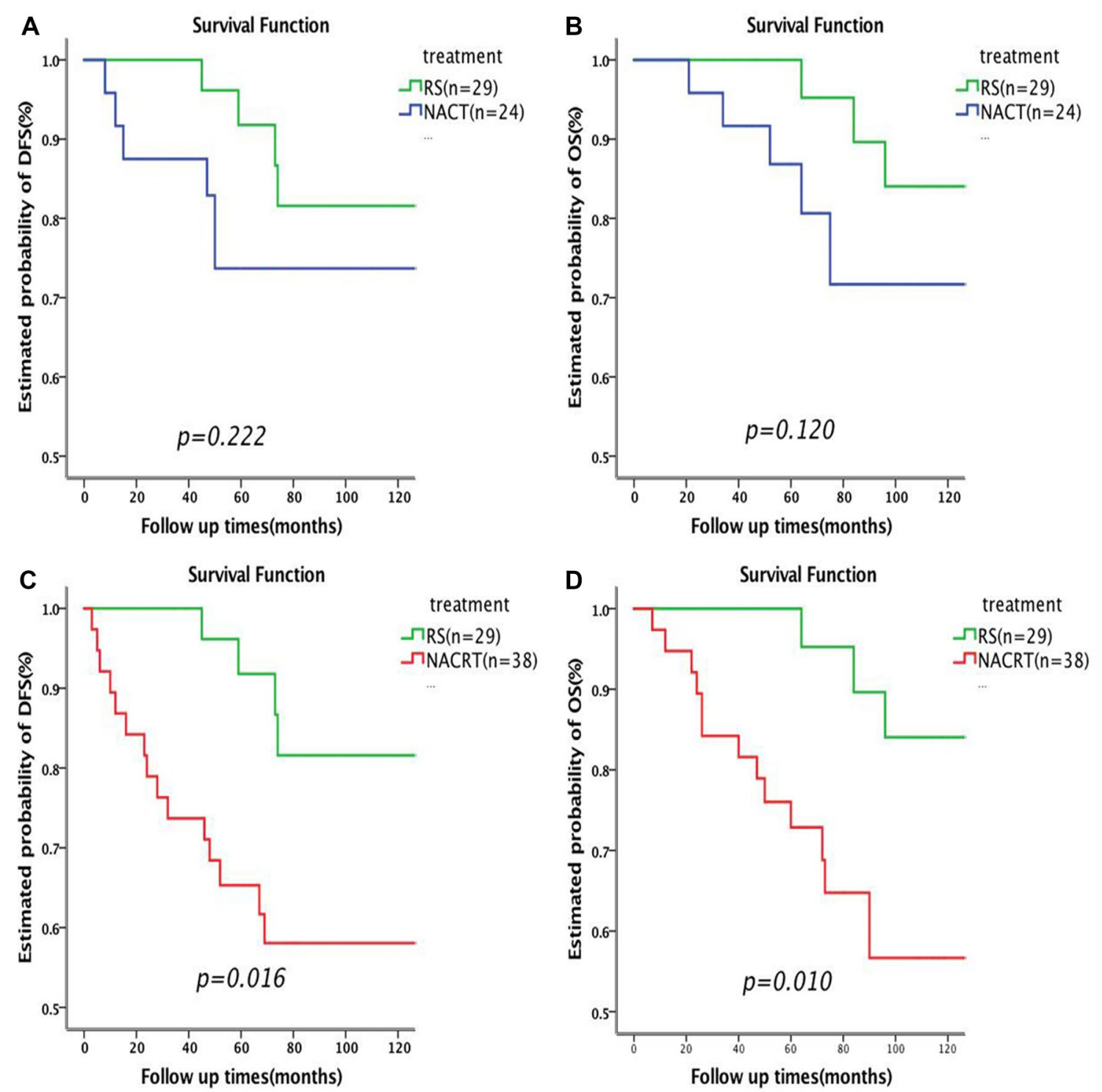

Fig. 2 Plot of Kaplan-Meier disease free survival (a) and overall survival (b) for the NACT + RS group compared with RS alone group and for disease free survival (c) and overall survival (d) for the

adenocarcinoma, or clear cell adenocarcinoma. The biological behavior of cervical AC must also be taken into account. Several studies have investigated survival outcomes in cervical AC to determine more appropriate treatment approaches. One possibility is NACT before CCRT. The use of NACT before CCRT followed by adjuvant therapy in patients with AC histology has been reported. Tang et al. compared NACT before CCRT followed by adjuvant therapy with CCRT alone in 880 patients with stages IIB-IVA AC and observed that sandwich chemotherapy is more effective and safe [30].

Patients in the NACRT + RS group who achieved pOR with neoadjuvant treatment still had poor prognosis (Fig. 3c, d). However, in several retrospective studies, neoadjuvant

NACRT + RS group compared with RS alone group. NACT neoadjuvant chemotherapy, $R S$ radical surgery, NACRT neoadjuvant chemoradiation therapy

brachytherapy and chemotherapy followed by RS for stage IB2 and IIA cervical cancer patients had no obvious inferiority to NACT + RS [31, 32]. Additionally, Vízkeleti et al. observed that the postoperative response rate in patients who received preoperative intrauterine brachytherapy was higher than that in patients who received RS alone $(P=0.03)$, and the rate of positive surgical margins was significantly lower $(P=0.02)$ [33]. This suggests that the underlying reason for our results is that radiotherapy and neoadjuvant brachytherapy reduce the pathological response in cervical AC.

This study has some limitations. We only included a small number of patients, and the retrospective analysis is not sufficiently rigorous to settle the question of the effects 

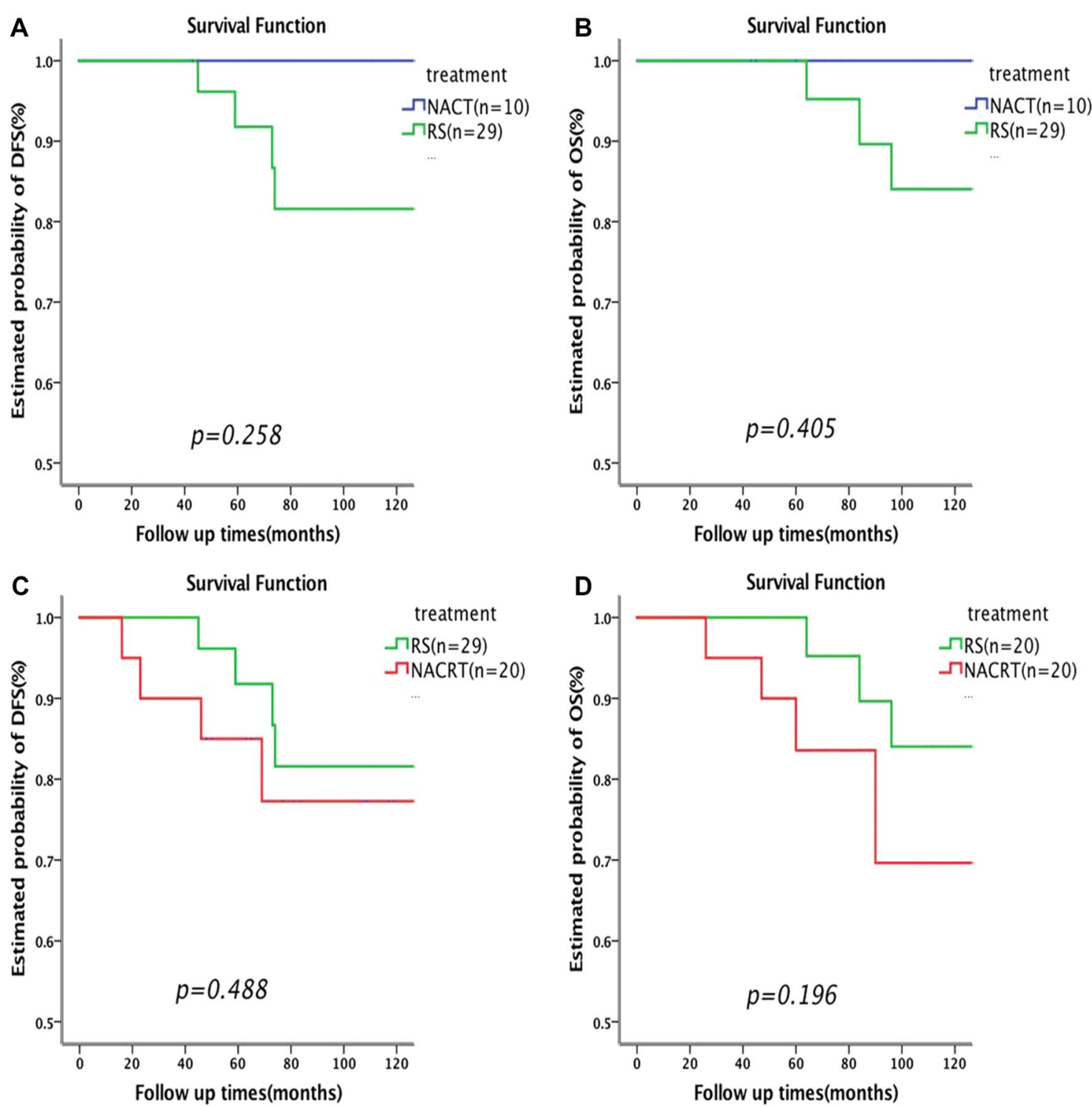

Fig. 3 Plot of Kaplan-Meier estimates for disease free survival (a) and overall survival (b) for the patients with optimal response in the NACT + RS group compared to the RS group and for disease free survival (c) and overall survival (d) for the patients with opti-

mal response in the NACRT + RS group compared to the RS group. $N A C T$ neoadjuvant chemotherapy, $R S$ radical surgery, NACRT neoadjuvant chemoradiation therapy
Table 6 Multivariate analysis of recurrence-free survival and overall survival

\begin{tabular}{|c|c|c|c|c|}
\hline \multirow[t]{2}{*}{ Varient } & \multicolumn{2}{|l|}{ DFS } & \multicolumn{2}{|l|}{ OS } \\
\hline & HR $(95 \%$ CI $)$ & $P$ & HR $(95 \%$ CI $)$ & $P$ \\
\hline Age (years) & $1.901(0.465-7.774)$ & 0.371 & $1.881(0.327-10.820)$ & 0.479 \\
\hline Tumor size $(\mathrm{cm})$ & $0.915(0.242-3.452)$ & 0.895 & $0.459(0.104-2.026)$ & 0.304 \\
\hline Histological grade & NA & 0.135 & NA & 0.134 \\
\hline Lymphnode metastasis & $0.223(0.060-0.827)$ & 0.025 & $0.088(0.017-0.470)$ & 0.004 \\
\hline Stage & $0.621(0.149-2.590)$ & 0.513 & $0.401(0.073-2.191)$ & 0.291 \\
\hline Depth of stromal invasion & $0.885(0.272-2.885)$ & 0.840 & $2.274(0.529-9.785)$ & 0.270 \\
\hline Treatment & $0.385(0.106-1.407)$ & 0.149 & $0.248(0.045-1.360)$ & 0.108 \\
\hline Respond of neoadjuvant therapy & $3.662(0.754-17.784)$ & 0.107 & $5.299(0.767-36.612)$ & 0.091 \\
\hline
\end{tabular}

$D F S$ disease free survival, $O S$ overall survival, $C I$ confidence interval, $H R$ hazard radio, $N A$ not available 
of neoadjuvant therapy in IB2/IIA2 cervical cancer. Studies with larger sample sizes and multicenter prospective randomized studies are needed to further determine the role of preoperative adjuvant therapy.

In conclusion, there was no significant difference in survival between cervical AC patients who received $\mathrm{NACT}+\mathrm{RS}$ and those who received RS alone. Patients who responded to NACT had favorable prognosis, which may suggest that there are subgroups in which neoadjuvant therapy may be beneficial. No survival benefit was observed in the NACRT group, even in responders.

Acknowledgements The authors would like to thank all the members of the Jing Wang laboratory.

Author contributions OYPL summarized available literature and drafted the paper. CJT, GL, and LS collected and analyzed the clinical data. WJ and WNY edited the paper and supervised the research group. All authors read and approved the final manuscript.

Funding This work was supported by the Clinical Research Center in Gynecologic Cancer, Hunan Cancer Hospital (QH201908), and the National Natural Science Foundation of China (81972836,2016YFC1303703, 2018JJ6109), and the Foundation from Social development science and technology division (2018SK2121, kq1801104), and Hunan Provincial Health Commission (20201487).

\section{Compliance with ethical standards}

Conflict of interest The authors declare that they have no competing interests.

Ethical approval The study was approved by the Hunan Cancer Hospital ethics committee \{project number: 2015[01] \} and the Chinese Clinical Trial Registry (registration number: ChiCTR1800018931).

Open Access This article is licensed under a Creative Commons Attribution 4.0 International License, which permits use, sharing, adaptation, distribution and reproduction in any medium or format, as long as you give appropriate credit to the original author(s) and the source, provide a link to the Creative Commons licence, and indicate if changes were made. The images or other third party material in this article are included in the article's Creative Commons licence, unless indicated otherwise in a credit line to the material. If material is not included in the article's Creative Commons licence and your intended use is not permitted by statutory regulation or exceeds the permitted use, you will need to obtain permission directly from the copyright holder. To view a copy of this licence, visit http://creativecommons.org/licenses/by/4.0/.

\section{References}

1. Bray F et al (2018) Global cancer statistics 2018: GLOBOCAN estimates of incidence and mortality worldwide for 36 cancers in 185 countries. CA Cancer J Clin 68(6):394-424

2. Young RH, Clement PB (2002) Endocervical adenocarcinoma and its variants: their morphology and differential diagnosis. Histopathology 41:185-207
3. Bray F et al (2005) Incidence trends of adenocarcinoma of the cervix in 13 European countries. Cancer Epidemiol Biomarkers Prev 14(9):2191-2199

4. Adegoke O, Kulasingam S, Virnig B (2012) Cervical cancer trends in the United States: a 35-year population-based analysis. J Womens Health (Larchmt) 21(10):1031-1037

5. Galic V et al (2012) Prognostic significance of adenocarcinoma histology in women with cervical cancer. Gynecol Oncol 125(2):287-291

6. Sedlis A, Brian BN, Rotman MZ, Lentz SS, Muderspach LI, Zaino RJ (1999) A randomized trial of pelvic radiation therapy versus no further therapy in selected patients with stage IB carcinoma of the cervix after radical hysterectomy and pelvic lymphadenectomy: a gynecologic oncology group study. Gynecol Oncol 73(2):177-183

7. Yokoi E, Mabuchi S, Takahashi R, Matsumoto Y, Kuroda H, Kozasa K, Kimura T (2017) Impact of histological subtype on survival in patients with locally advanced cervical cancer that were treated with defnitive radiotherapy: adenocarcinoma/adenosquamous carcinoma versus squamous cell carcinoma. J Gynecol Oncol 28(2):e19

8. Hu K et al (2018) Comparison of treatment outcomes between squamous cell carcinoma and adenocarcinoma of cervix after definitive radiotherapy or concurrent chemoradiotherapy. Radiat Oncol 13(1):249

9. Chen $\mathrm{H}$ et al (2008) Clinical efficacy of modified preoperative neoadjuvant chemotherapy in the treatment of locally advanced (stage IB2 to IIB) cervical cancer: a randomized study. Gynecol Oncol 110(3):308-315

10. Katanyoo K, Sanguanrungsirikul S, Manusirivithaya S (2012) Comparison of treatment outcomes between squamous cell carcinoma and adenocarcinoma in locally advanced cervical cancer. Gynecol Oncol 125(2):292-296

11. Gien LT, Beauchemin MC, Thomas G (2010) Adenocarcinoma: a unique cervical cancer. Gynecol Oncol 116(1):140-146

12. Bader AA et al (2006) Is intraoperative frozen section analysis of pelvic lymph nodes accurate after neoadjuvant chemotherapy in patients with cervical cancer? Gynecol Oncol 103(1):106-112

13. Gong L et al (2012) Clinical evaluation of neoadjuvant chemotherapy followed by radical surgery in the management of stage IB2-IIB cervical cancer. Int J Gynecol Obstet 117(1):23-26

14. Katsumata $\mathrm{N}$ et al (2013) Phase III randomised controlled trial of neoadjuvant chemotherapy plus radical surgery vs radical surgery alone for stages IB2, IIA2 and IIB cervical cancer: a Japan clinical oncology group trial (JCOG 0102). Br J Cancer 108(10):1957-1963

15. Kim HS et al (2013) Efficacy of neoadjuvant chemotherapy in patients with FIGO stage IB1 to IIA cervical cancer: an international collaborative meta-analysis. Eur J Surg Oncol (EJSO) 39(2):115-124

16. Landoni $\mathrm{F}$ et al (2014) Is there a role for postoperative treatment in patients with stage Ib2-IIb cervical cancer treated with neo-adjuvant chemotherapy and radical surgery? An Italian multicenter retrospective study. Gynecol Oncol 132(3):611-617

17. Rydzewska L, Tierney J, Vale CL, Symonds PR (2010) Neoadjuvant chemotherapy plus surgery versus surgery for cervical cancer (review). Cochrane Database Syst Rev 12(1):CD007406

18. Piver MS, Rutledge F, Smith JP (1974) Five classes of extended hysterectomy for women with cervical cancer. Obstet Gynecol 44(2):265-272

19. Chuang LT et al (2016) management and care of women with invasive cervical cancer: American society of clinical oncology resource-stratified clinical practice guideline. J Glob Oncol 2(5):311-340

20. Ferrandina $G$ et al (2018) Dose-dense paclitaxel/carboplatin as neo-adjuvant chemotherapy followed by radical surgery in locally 
advanced cervical cancer: a prospective phase II study. Cancer Chemother Pharmacol 83(3):431-438

21. Kenter G, Greggi S, Vergote I, Katsaros D, Kobierski J, Massuger L, van Doorn HC, Landoni F, Van Der Velden J, Reed NS, Coens C, van Luijk I, Ottevanger PB, Casado A (2019) Results from neoadjuvant chemotherapy followed by surgery compared to chemoradiation for stage Ib2-IIb cervical cancer, EORTC 55994. J Clin Oncol 37(15_suppl):5503

22. Gupta $S$ et al (2018) Neoadjuvant chemotherapy followed by radical surgery versus concomitant chemotherapy and radiotherapy in patients with stage IB2, IIA, or IIB squamous cervical cancer: a randomized controlled trial. J Clin Oncol 36(16):1548-1555

23. Ye Q, Yuan HX, Chen HL (2013) Responsiveness of neoadjuvant chemotherapy before surgery predicts favorable prognosis for cervical cancer patients: a meta-analysis. J Cancer Res Clin Oncol 139(11): 1887-1898

24. Landoni F, Sartori E, Maggino T, Zola P, Zanagnolo V, Cosio S, Ferrari F, Piovano E, Gadducci A (2013) Is there a role for postoperative treatment in patients with stage Ib2-IIb cervical cancer treated with neo-adjuvant chemotherapy and radical surgery? An Italian multicenter retrospective study. Gynecol Oncol 132(3):611-617

25. Sun H et al (2018) Adjuvant chemotherapy after surgery can improve clinical outcomes for patients with IB2-IIB cervical cancer with neoadjuvant chemotherapy followed by radical surgery. Sci Rep 8(1):1-8

26. Baalbergen A (2004) Prognostic factors in adenocarcinoma of the uterine cervix. Gynecol Oncol 92(1):262-267

27. Irie $\mathrm{T}$ et al (2000) Prognosis and clinicopathological characteristics of Ib-IIb adenocarcinoma of the uterine cervix in patients who have had radical hysterectomy. Eur J Surg Oncol 26(5):464-467
28. Mabuchi S et al (2012) Impact of histological subtype on survival of patients with surgically-treated stage IA2-IIB cervical cancer: adenocarcinoma versus squamous cell carcinoma. Gynecol Oncol 127(1):114-120

29. Ryu SY et al (2013) Intermediate-risk grouping of cervical cancer patients treated with radical hysterectomy: a Korean gynecologic oncology group study. Br J Cancer 110(2):278-285

30. Tang $\mathbf{J}$ et al (2012) Chemoradiation and adjuvant chemotherapy in advanced cervical adenocarcinoma. Gynecol Oncol 125(2):297-302

31. Pearcey RG et al (1988) The value of pre-operative intracavitary radiotherapy in patients treated by radical hysterectomy and pelvic lymphadenectomy for invasive carcinoma of the cervix. Clin Radiol 39(1):95-98

32. Ma Y et al (2018) Neoadjuvant brachytherapy and chemotherapy followed by radical surgery for stage IB2 and IIA cervical cancer: a retrospective comparison with chemoirradiation. Mol Clin Oncol 8(4):623-627

33. Vízkeleti J et al (2014) Pathologic complete remission after preoperative high-dose-rate brachytherapy in patients with operable cervical cancer: preliminary results of a prospective randomized multicenter study. Pathol Oncol Res 21(2):247-256

Publisher's Note Springer Nature remains neutral with regard to jurisdictional claims in published maps and institutional affiliations. 